\title{
Visualization for Secure Coding in C
}

\author{
Extended Abstract
}

\author{
James Walker, Jean Mayo, \\ Ching-Kuang Shene \\ Michigan Technological University \\ Houghton, MI \\ jwwalker,jmayo,shene@mtu.edu
}

\author{
Steve Carr \\ Western Michigan University \\ Kalamazoo, MI \\ steve.carr@wmich.edu
}

\begin{abstract}
This paper describes a pedagogical system to visualize program execution. ${ }^{1}$ The visualization is designed to help students understand how to develop more secure and robust $\mathrm{C}$ programs. The system provides several perspectives on the execution including: the values of registers and the logical address space, a call graph, the file descriptor and inode tables, and the handling of sensitive data like passwords and keys. These visualizations are designed to help students understand fundamental concepts such as: buffer overflows, integer overflows, proper handling of sensitive data and application of the principle of least privilege in several contexts including file operations, secure SUID programming, and use and management of the process environment.
\end{abstract}

\section{Keywords}

Security Education; Program Execution; Visualization

\section{OVERVIEW}

Many program vulnerabilities are introduced into C programs through poor understanding of the program's layout in memory and its execution. We have developed a visualization system to help students make the connection between their $\mathrm{C}$ code and its execution. The system was designed to teach secure coding in $\mathrm{C}$, but can be useful in many courses including courses on $\mathrm{C}$, systems programming, concurrent computing, and operating systems.

\section{VISUALIZATIONS}

The system currently provides four perspectives: Program Address Space (PA), Call Graph (CG), File Operations (FO) and Sensitive Data (SD). The system takes input from dynamic analysis using Pintool[1]. The analysis produces a

\footnotetext{
${ }^{1}$ This work has been supported by the National Science Foundation under grants DUE-1245310, DGE-1523017, IIS1456763 , and IIS-1455886.
}

Permission to make digital or hard copies of part or all of this work for personal or classroom use is granted without fee provided that copies are not made or distributed for profit or commercial advantage and that copies bear this notice and the full citation on the first page. Copyrights for third-party components of this work must be honored. For all other uses, contact the owner/author(s).

ITiCSE' 17 July 03-05, 2017, Bologna, Italy

(C) 2017 Copyright held by the owner/author(s).

ACM ISBN 978-1-4503-4704-4/17/07.

DOI: http://dx.doi.org/10.1145/3059009.3072990 sequence of events that are processed by the visualizations. A student can step through an execution and view one or multiple visualizations at once. Source code is displayed and events are linked to the corresponding line of source code.

The PA visualization depicts the values of registers and the program address space. Students may expand and collapse individual activation records. A user may choose among multiple levels of detail. For example, data related to the use of shared libraries can be hidden. The PA visualization clearly depicts buffer overflows, integer overflows and other memory errors.

The CG visualization depicts the sequence of function calls made during an execution. In its least complex form, the hierarchy of processes created using fork() is displayed. The user can freely expand and collapse the sequence of calls. This visualization intends to help students understand and identify sequences of actions that affect program security. This includes execution with unnecessary privileges and improper handling of sensitive data.

The FO visualization shows kernel structures commonly used to maintain the UNIX file abstraction. This helps students to understand the impact of parameters to the open() system call and to understand how parent and child interact through the file system following a call to fork() or exec().

The SD visualization teaches students how to protect sensitive data so that it never appears unencrypted on secondary storage. The visualization compares related actions in a student's program to a sequence of actions required to keep the data secure. The PA visualization can be used with the SD visualization to show students the correspondence between their code and the appearance of sensitive data in memory.

\section{CONCLUSIONS AND FUTURE WORK}

The system will be evaluated in classroom use in courses at several levels within our undergraduate curriculum. The visualization will be integrated into an IDE that identifies and explains security vulnerabilities as a student edits her $\mathrm{C}$ program. A module for visualizing the output from a taint analysis of $\mathrm{C}$ programs is under development.

\section{REFERENCES}

[1] Intel Software. Pin - a dynamic binary instrumentation tool. https://software.intel.com/en-us/articles/ pin-a-dynamic-binary-instrumentation-tool, 2017. [Online; accessed 10-Mar-2017]. 